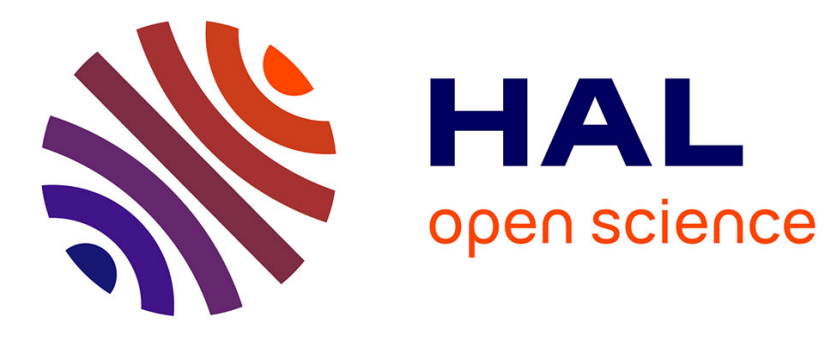

\title{
Designing virus-resistant networks: a game-formation approach
}

\author{
Stojan Trajanovski, Fernando Antonio Kuipers, Yezekael Hayel, Eitan \\ Altman, Piet van Mieghem
}

\section{- To cite this version:}

Stojan Trajanovski, Fernando Antonio Kuipers, Yezekael Hayel, Eitan Altman, Piet van Mieghem. Designing virus-resistant networks: a game-formation approach. IEEE 54th Annual Conference on Decision and Control (CDC), Dec 2015, Osaka, Japan. hal-01204628

\section{HAL Id: hal-01204628 \\ https://hal.inria.fr/hal-01204628}

Submitted on 24 Sep 2015

HAL is a multi-disciplinary open access archive for the deposit and dissemination of scientific research documents, whether they are published or not. The documents may come from teaching and research institutions in France or abroad, or from public or private research centers.
L'archive ouverte pluridisciplinaire HAL, est destinée au dépôt et à la diffusion de documents scientifiques de niveau recherche, publiés ou non, émanant des établissements d'enseignement et de recherche français ou étrangers, des laboratoires publics ou privés. 


\title{
Designing virus-resistant networks: a game-formation approach
}

\author{
Stojan Trajanovski ${ }^{1}$, Fernando A. Kuipers ${ }^{1}$, Yezekael Hayel ${ }^{2},{\text { Eitan } \text { Altman }^{3} \text {, and Piet Van Mieghem }}^{1}$
}

\begin{abstract}
Forming, in a decentralized fashion, an optimal network topology while balancing multiple, possibly conflicting objectives like cost, high performance, security and resiliency to viruses is a challenging endeavor. In this paper, we take a game-formation approach to network design where each player, for instance an autonomous system in the Internet, aims to collectively minimize the cost of installing links, of protecting against viruses, and of assuring connectivity.

In the game, minimizing virus risk as well as connectivity costs results in sparse graphs. We show that the Nash Equilibria are trees that, according to the Price of Anarchy (PoA), are close to the global optimum, while the worst-case Nash Equilibrium and the global optimum may significantly differ for small infection rate and link installation cost. Moreover, the types of trees, in both the Nash Equilibria and the optimal solution, depend on the virus infection rate, which provides new insights into how viruses spread: for high infection rate $\tau$, the path graph is the worst- and the star graph is the best-case Nash Equilibrium. However, for small and intermediate values of $\tau$, trees different from the path and star graphs may be optimal.
\end{abstract}

\section{INTRODUCTION}

Designing communication and computer networks requires balancing performance, security and cost investments, which makes network design complex. For instance, let us consider a host in a computer network that wants to route traffic to multiple other hosts. The host could directly connect to those other hosts, in this way increasing its expenses and being more susceptible to viruses, but also having a better and faster performance with minimum delays, rather than using intermediate hosts as relays. However, reducing the installation expenses (e.g., equipment costs) and suppressing potential viruses do not necessarily optimize together. Indeed, reducing the number of direct connections would reduce the cost and the host would be less vulnerable to viruses. However, even when being connected to few nodes with direct connections, if the neighbors are highly connected, the host would still be at a high risk of becoming infected by a virus. In practice, hosts often are autonomous, act independently and uncoordinated like in P2P networks [1], peering relations between Autonomous Systems [2], overlay networks [3], wireless [4] and mobile [5] networks, resource allocation [6], social networks [7] or the Internet [8], and they aim to optimize their own utility functions, which is not necessarily in accordance to the global optimum.

To study global network performance, the network formation game (NFG) framework [9] has been proposed.

\footnotetext{
${ }^{1}$ Delft University of Technology, Delft, The Netherlands (e-mails: \{S.Trajanovski, F.A.Kuipers, P.F.A.VanMieghem\}@ tudelft.nl).

${ }^{2}$ University of Avignon, Avignon, France and NYU, Polytechnic School of Engineering, Brooklyn, USA (e-mail: yezekael.hayel@univ-avignon.fr).

${ }^{3}$ INRIA, Sophia Antipolis, France (e-mail: eitan.altman@inria.fr).
}

Security and notably virus protection have not been taken into account in the NFG context, even though their importance is undisputed. In this paper, we develop an NFG framework by including virus protection and connectivity as key ingredients. Virus propagation will be modeled by the Susceptible-Infected-Susceptible (SIS) model [10]. We evaluate the effect of uncoordinated autonomous hosts versus the optimal solution by standard game theoretic concepts, such as the Nash Equilibria and the Prices of Anarchy and Stability.

We consider a game, called the Virus Spread-Cost (VSC) game, in which a node (player) in a non-cooperative communication network aims, at least cost, to minimize its chance of being infected by a virus, while still being able to route traffic to all the other nodes. Indeed, both the virus and cost objectives tend to thin-out the graph, but exploring how the hosts interconnect in such a sparse topology would help understand the process of virus spread better. Our key contributions are summarized as follows:

- In the VSC game, we find that a Nash Equilibrium always exists and is a tree, although not any tree is a Nash Equilibrium. The tree that is the worst-case Nash Equilibrium depends on the effective infection rate.

- A Nash Equilibrium in the VSC game is shown to be pairwise stable [11].

- For high effective infection rate $\tau$, the Price of Anarchy (PoA) in the VSC game is generally close to 1 , independent from the number of hosts, and is inversely proportional to the virus infection rate and the link installation cost. This implies that non-cooperative players still form a close-to-optimal topology for high $\tau$. On the other hand, the PoA may be very high for small effective infection rate $\tau$ and/or small installation cost.

This paper is organized as follows. Related work on game formation and network protection for virus spread is discussed in Section II. The SIS-virus spread model and the network-formation game-theory model are introduced in Section III. The Virus Spread-Cost (VSC) game formation is studied and analyzed in Section IV. Conclusions and directions for future work are given in Section V.

\section{RELATED WORK}

There is a large body of literature on game formation, which mostly minimizes a cost utility based on hopcount and the cost for installing links [8], [2], [12], [3], [13], [14], [15], [16], [17]. Fabrikant et al. [8] have studied the case, where a node's utility is a weighted sum of the installed links and the sum of hopcounts to each node in an undirected graph. The follow-up work by Albers et al. [12] resolves some open 
questions from [8]. Chun et al. [3] have conducted extensive simulations on this game formation. A game formation problem involving hopcounts and costs, applied to P2P networks has been considered by Mascibroda et al. [13]. Nahir et al. [15] have considered similar NFG problems in directed graphs. Coalition and bilateral agreements between players in NFG and game-theory in general have been considered in [18], [5], [19], [2]. In order to evaluate "the goodness" of the equilibria, the prices of anarchy and stability [14], [20] have been used.

Epidemic spread and virus-spread protection in networks have been studied in the past [21], [22], [10], [23], [24], [25]. Although game theory has been used in modeling the virus spread suppression and network immunization [26], [27], [28], [29], [30], [31], security aspects and the resilience to virus spread have not been accounted for in the network formation game (NFG) framework. Our work extends, with virus spread, the related work from the NFG framework [8], [12], [15].

\section{MODELS AND PROBLEM STATEMENTS}

\section{A. Virus-spread model}

The spread of viruses in communication and computer networks can be described using virus-spread epidemic models [21], [10], [22]. We consider the Susceptible-InfectedSusceptible (SIS) model,

$$
\frac{d v_{i}(t)}{d t}=\beta\left(1-v_{i}(t)\right) \sum_{j=1}^{N} a_{i j} v_{j}(t)-\delta v_{i}(t)
$$

where $v_{i}(t)$ is the infection probability of node $i$ at time $t$, for all nodes $i \in\{1,2, \ldots, N\}$. If a link is present between nodes $i$ and $j$, then $a_{i j}=1$, otherwise $a_{i j}=0$, where the elements $a_{i j}$ form the $N \times N$ adjacency matrix. In (1), a host with a virus can infect its direct healthy neighbors with rate $\beta$, while an infected host can be cured at rate $\delta$, after which the node becomes healthy, but susceptible again to the virus. The infection probability of node $i$ in the metastable regime, denoted by $v_{i \infty}$, where $\frac{d v_{i}(t)}{d t}=0$ and $v_{i \infty} \neq 0$, is deduced from (1) as

$$
0=\beta\left(1-v_{i \infty}\right) \sum_{j=1}^{N} a_{i j} v_{j \infty}-\delta v_{i \infty}
$$

or re-written to

$$
v_{i \infty}=1-\frac{1}{1+\tau \sum_{j=1}^{N} a_{i j} v_{j \infty}}
$$

where $\tau=\frac{\beta}{\delta}$ is called the effective infection rate. The epidemic threshold $\tau_{c}$ is defined as a value of $\tau$, such that $v_{i \infty}>0$ if $\tau>\tau_{c}$, and otherwise $v_{i \infty}=0$ for all $i \in\{1,2, \ldots, N\}$.

The network stabilizes and stays long enough in the metastable regime, before going to the trivial stable regime where $v_{i}=0$ for $\forall i$. This is the main motivation to focus on the virus risk minimization in the metastable regime, given in (2).

\section{B. Game-formation model}

We study two novel network formation games, where each player $i$ (a node in the network) aims to minimize its own cost function $J_{i}$, and the social cost $J$ is defined as $J=$ $\sum_{i=1}^{N} J_{i}$. Specifically, the optimal social cost $\min J$ is the optimal (minimum) sum of all players' costs. We look for the existence, uniqueness, and characterization of (pure) Nash Equilibria $^{1}$. The Price of Anarchy (PoA) and the Price of Stability $(P o S)$ are defined as the ratio of social cost in the worst-case Nash Equilibrium (with $\max J$ ) and the optimal social cost, and the ratio of the social cost in the best-case Nash Equilibrium (with $\min J$ ) and the optimal social cost, respectively:

$$
\operatorname{PoA}=\frac{J(\text { worst } \mathrm{NE})}{\min J}, \operatorname{PoS}=\frac{J(\text { best } \mathrm{NE})}{\min J}
$$

PoA is an efficiency measure and it shows how bad selfish playing is, when compared to the global optimum. PoS, on the other hand, reflects the best possible performance without coordination when compared to the global optimum.

The network to be designed, is empty and every node in the network is a player. We assume the cost of building one (communication) link between two nodes is fixed. Every player $i$ can install a link from itself to another node $j$. A new link between $i$ and $j$ can be used by both players, but only one of them pays for the cost, like often assumed in NFG models [8], [12], [3]. Sometimes, creating a link involves two players, in which case the equilibrium has to be robust against deviation of any pair of the two players. Although the obtained metrics might differ [2], there is no big conceptual difference in our methodology with the case that the cost is shared. We will therefore only consider "how robust" the obtained Nash Equilibrium is in terms of players' deviations for the incident links to them, but not installed by them. This notion of pairwise stability has been proposed for network formation games in [11]. The pairwise stability determines if each player is robust to one-link deviations (not necessarily installed by him), from a unilateral move. The concept of pairwise Nash Equilibrium (PNE) in this type of game has to take into account simultaneous moves. A PNE exists if and only if both nodes are interested in having a link between them.

We study a Virus Spread-Cost (VSC) network formation game, where the utility $J_{i}$ of player $i$ is a weighted sum of the infection probabilities $v_{i \infty}$ in the metastable state and the cost $k_{i}$ of all the links that $i$ installs, under the condition that player $i$ should be able to reach all the nodes in the network. The utility of player $i$ is given by:

$$
J_{i}= \begin{cases}\alpha \cdot k_{i}+v_{i \infty}, & \text { if } i \text { can reach all the nodes, } \\ \infty, & \text { otherwise. }\end{cases}
$$

Function $J_{i}$ involves the cost $k_{i}$ of installing all the links from node $i$, weighted by a coefficient $\alpha$. This game can be written as a coupled constraint game [32], where the decisions of all the players have the common constraint that

\footnotetext{
${ }^{1} \mathrm{~A}$ Nash Equilibrium is the state of the players' network strategies, where none of the players can reduce its cost by unilaterally changing its strategy.
} 
the graph is fully connected. The cost $k_{i}$ is not larger than the degree $d_{i}$ of node $i\left(k_{i} \leq d_{i}\right)$, because $d_{i}$ counts all the links that $i$ installs, but also all the links that other nodes install to $i$ and $i$ "benefits" from. A node should be resistant to viruses, which is expressed by the infection probability $v_{i \infty}$ of node $i$ in the metastable state given in (2). The virus and cost both contribute to having a sparse topology. How the hosts are interconnected in such a sparse topology would explain the virus spread process and its implications on the network design. These aspects are covered in Section IV.

\section{VIRUS SPREAD-COST}

In the Virus Spread-Cost game from (4), the social cost $J$ for the whole network is given by

$$
J=\alpha \sum_{i=1}^{N} k_{i}+\sum_{i=1}^{N} v_{i \infty}=\alpha L+\sum_{i=1}^{N} v_{i \infty}
$$

if the graph is connected, otherwise $J=\infty$, where $L=$ $\sum_{i=1}^{N} k_{i}$ is the number of links in the network. To find Nash Equilibria, we introduce the following Lemma 1.

Lemma 1. The infection probability $v_{i \infty}(G)$ of each node $i$ in the metastable state in network $G$ is not bigger than the infection probability $v_{i \infty}(G+l)$ of node $i$ in the metastable state in network $G+l$ obtained by adding a link $l$ to $G$.

Proof. The newly added link $l=(a, b)$ is between nodes $a$ and $b$. We make use of the canonical infinite form [10],

$$
v_{i \infty}=1-\frac{1}{1+\tau d_{i}-\tau \sum_{j=1}^{N} \frac{a_{i j}}{1+\tau d_{j}-\tau \sum_{k=1}^{N} \frac{a_{j k}}{1+\tau d_{k}-} \cdot}} .
$$

Expression (6) for $v_{i \infty}(G+l)$ has all the terms the same as in $v_{i \infty}(G)$, except where $a$ and $b$ are neighbors in the canonical representation. The last implies that its contribution is a part that is the same as in $v_{i \infty}(G)$ plus the term:

$$
\begin{aligned}
& \tau\left(d_{a}+1\right)-\frac{1}{\tau\left(d_{b}+1\right)-\ldots}-U \\
& =\tau d_{a}-U+\tau\left(1-\frac{1}{\tau\left(d_{b}+1\right)-\ldots}\right),
\end{aligned}
$$

where $d_{a}$ and $d_{b}$ are the degrees of $a$ and $b$ in $G$, while $U$ is the remaining part in the canonical form at the specific depth. In (7), the term $\tau\left(1-\frac{1}{\tau\left(d_{b}+1\right)-\ldots}\right)$ is positive and $U$ decreases. Therefore, the whole term in (7) increases, which implies that $v_{i \infty}(G+l)>v_{i \infty}(G)$ also increases for each node $i$.

A. Optimal social cost, Nash Equilibria and the Price of Anarchy

We start by looking into the possible Nash Equilibria. The following Theorem 1 narrows down the possibilities.

Theorem 1. If a Nash Equilibrium is achieved, then the constructed graph is a tree.

Proof. Let us first assume that a Nash Equilibrium is reached. First, if $G$ is connected and each node can reach every other node, then changing a strategy of player (node) $i$ from the current one to investing in an extra link, will increase both the cost (by 1 , scaled by $\alpha$ ) and $v_{i \infty}$ (by Lemma 1). Hence, unilaterally changing by investing in an extra link is not beneficial for a node. (Its cost utility is increased.)

We now assume that $G$ is not a tree. Then, there is at least one cycle in this graph. If a node $i$ in that cycle changes its strategy from investing in a link in that cycle to not investing, the cost is decreased by 1 (weighted by $\alpha$ ) and all the other nodes in the graph are still reachable from $i$. Moreover, by not investing in that link, player $i$ decreases its infection probability $v_{i \infty}$ in the metastable state, according to Lemma 1 . Hence, by unilaterally changing its strategy, player $i$ decreases its cost utility $J_{i}$, which is in contradiction with the Nash Equilibrium.

Observation 1. A Nash Equilibrium is achieved for both the star graph and the path graph, but not all trees are Nash Equilibria.

Proof. Let us consider a star graph, where all the links are installed by the root node as shown in Figure 1a. (A link is installed and paid by the node marked with $p$.) The root node cannot unilaterally decrease its cost, because cutting at least one of its installed links would disconnect, while installing a link from a leaf node $i$ would increase both $k_{i}$ and $v_{i \infty}$ (Lemma 1). Hence, the star graph is a Nash Equilibrium.

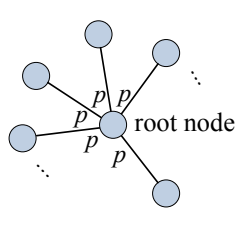

(a) $\operatorname{Star} K_{1, N-1}$.

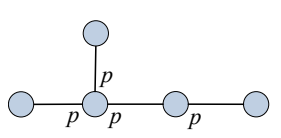

(c) Tree $T^{\prime}$.

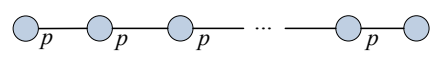

(b) Path $P_{N}$.

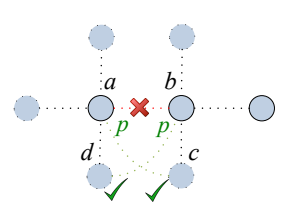

(d) Tree $T^{\prime \prime}$.

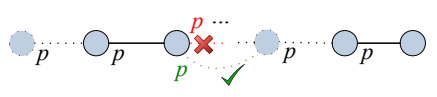

(e) Re-wiring increases $v_{i \infty}$.

Fig. 1: A link is installed by the end-node marked with $p$. Trees in (a), (b), and (c) are Nash Equilibria. (d) Tree $T^{\prime \prime}$ cannot be a Nash Equilibrium.

Let us now assume that a path graph is constructed, such that a leaf invests for a link to the next node, and every next node along the path invests for a link to the next node all 
the way to the other leaf (Figure 1b). At the end, $(N-1)$ nodes invest in exactly one link and one of the leaves does not invest in installing a link. Similarly as for a star graph, none of the nodes can unilaterally decrease its cost by just installing extra links or cutting some of them. Finally, just a "re-wiring" from one of the nodes by re-directing its installed links to another node is in order. In such a case, if node $i$ "re-wires" its installed link to another node, then $J_{i}$ would not decrease. 1) If it is installed to one of the leaves, such that the graph is connected, we end up with an isomorphic graph, where the position of $i$ is the same as in the initial graph, so $J_{i}$ stays the same. 2) If $i$ "re-wires" to one of the other nodes $j$ (w.l.o.g., $i<j$ ) as visualized in Figure 1e, $v_{i \infty}$ would increase because $i$ would have the same degree, but its "new neighbor" would have a degree 3 instead of 2. The degree of $j$ increases by 1 to 3 and the degree of $(i+1)$ decreases by 1 to 1 , while all the other degrees remain the same. Moreover, $i$ would be equally close to any of the nodes "behind" $\{1, \ldots, i-1\}$, closer to the nodes "at the end" $\{j+1, \ldots, N\}$ and equally close to the nodes in the set $\{i+1, \ldots, j-1\}$, but just in a reverse order. Therefore, the path graph is also a Nash Equilibrium.

There are also other trees that are Nash Equilibria (e.g., $T^{\prime}$ given in Figure 1c), but not all the trees are Nash Equilibria. For example, the tree given in Figure 1d. Here, whomever pays for the "central" link between $a$ and $b$, can reduce its cost utility by "re-wiring" to $c$ or $d$.

We proceed by introducing Lemmas 2 and 3 to study the worst- and best-case Nash Equilibria.

Lemma 2. Assume that $G$ is a tree with a degree sequence $\left[d_{1}, d_{2}, \ldots, d_{N}\right]$ and $G^{\prime}$ is a tree with a degree sequence $\left[d_{1}, d_{2}, \ldots, d_{a-1}, d_{a}-1, \ldots, d_{b-1}, d_{b}+1, \ldots, d_{N}\right]$ that is the same as $G$, except for nodes $a$ and $b$. If $N-1 \geq d_{a} \geq$ $d_{b}+2 \geq 3$, then for sufficiently high effective infection rate $\tau, J(G)<J\left(G^{\prime}\right)$.

Proof. The number of links in any tree with $N$ nodes is $L=N-1$, hence the contribution from the costs of installed links is constant and the cost $J$ for node $i$ solely depends on the sum $y(\tau)=\sum_{i=1}^{N} v_{i \infty}(\tau)$. For sufficiently high $\tau$, the Laurent expansion for this sum in $\tau^{-1}$ is [24]

$$
y(\tau)=\sum_{i=1}^{N} v_{i \infty}(\tau)=N-\sum_{i=1}^{N} \frac{1}{\tau d_{i}}+O\left(\tau^{-2}\right) .
$$

Using (8), we consider the difference

$$
\begin{aligned}
& y_{G^{\prime}}(\tau)-y_{G}(\tau)=-\frac{1}{\tau\left(d_{a}-1\right)}-\frac{1}{\tau\left(d_{b}+1\right)}+\frac{1}{\tau d_{a}}+\frac{1}{\tau d_{b}} \\
& +O\left(\tau^{-2}\right)=\frac{1}{\tau}\left(\frac{1}{d_{b}\left(d_{b}+1\right)}-\frac{1}{d_{a}\left(d_{a}-1\right)}\right)+O\left(\tau^{-2}\right) .
\end{aligned}
$$

For sufficiently high $\tau(\tau>2)$, the value of $O\left(\tau^{-2}\right)$ tends to zero. Second, $d_{a}>d_{a}-1 \geq d_{b}+1>d_{b}$, therefore $\frac{1}{d_{b}\left(d_{b}+1\right)}>\frac{1}{d_{a}\left(d_{a}-1\right)}$, that in (9), gives $y_{G^{\prime}}(\tau)-y_{G}(\tau)>0$. Hence, $J(G)<J\left(G^{\prime}\right)$.
Lemma 3. Assume that $G$ is a tree with a degree sequence $\left[d_{1}, d_{2}, \ldots, d_{N}\right]$ and $G^{\prime}$ is a tree with a degree sequence $\left[d_{1}, d_{2}, \ldots, d_{a-1}, d_{a}+1, \ldots, d_{b-1}, d_{b}-1, \ldots, d_{N}\right]$ that is the same as $G$, except for nodes $a$ and $b$. If $N-2 \geq d_{a} \geq$ $d_{b} \geq 2$, then for sufficiently high effective infection rate $\tau$, $J(G)>J\left(G^{\prime}\right)$.

Proof. Follows analogously to the proof of Lemma 2.

Theorem 2. For sufficiently high effective infection rate $\tau$, the optimal cost and the best-case Nash Equilibrium are achieved by the star graph $K_{1, N-1}$, while the worst-case Nash Equilibrium is achieved for the path graph $P_{N}$,

$$
J\left(K_{1, N-1}\right) \leq J \leq J\left(P_{N}\right)
$$

Proof. Let us assume that in $G$, there are two nodes $i$ and $j$ for which $d_{i}-1 \geq d_{j}+1$. Replacing the degrees $d_{i}$ and $d_{j}$ by degrees $\left(d_{i}-1\right)$ and $\left(d_{j}+1\right)$, respectively, is possible, for instance by "re-wiring." The cost is increased and a tree $G_{1}$ is obtained. We continue with this procedure until there are at least two nodes with this property, obtaining consecutive trees $G_{2}, G_{3}, \ldots, G_{t}$, where in $G_{t}$ there are no such two pairs. The only possible tree with this property is the path graph $P_{N}$, hence $G_{t}=P_{N}$. Based on Lemma 2, and the fact that $G_{0}=G$ is any tree $J(G)<J\left(G_{1}\right)<\ldots<J\left(P_{N}\right)$.

Second, let us now assume that in $G$, there is a pair $(i, j)$ of nodes for which $N-2 \geq d_{i} \geq d_{j} \geq 2$. Replacing the degrees $d_{i}$ and $d_{j}$ by degrees $\left(d_{i}+1\right)$ and $\left(d_{j}-1\right)$, respectively (possible by "re-wiring"), the utility is decreased and a tree $G_{1}$ is obtained. We continue with this procedure until, as in the previous paragraph, we obtain a tree not having two nodes with the property $N-2 \geq d_{i} \geq d_{j} \geq 2$. Similarly, a star graph $K_{1, N-1}$ is obtained with minimum $J$.

However, what would be the optimum social cost, and the worst- and best-case Nash Equilibria highly depends on the effective infection rate $\tau$.

Theorem 3. For low values of the effective infection rate $\tau \leq \tau_{c}\left(K_{1, N-1}\right)$, the social cost $J(T)=\alpha(N-1)$ for any tree $T$. For values of the epidemic threshold $\tau_{c}\left(K_{1, N-1}\right)<$ $\tau \leq \tau_{c}\left(P_{N}\right)$, the social cost $J(T)>J\left(P_{N}\right)=\alpha(N-1)$ for any tree $T$.

Proof. We consider a spectral approach. The probabilities of a node in the graph being infected are non-zero and $y(\tau)>0$ if $\tau>\tau_{c}=\frac{1}{\lambda_{1}}$, where $\lambda_{1}$ is the largest eigenvalue of the adjacency matrix in the graph [21]. For $\tau<\frac{1}{\lambda_{1}}, y(\tau)=0$.

Lovász and Pelikán [33] ordered all the trees with $N$ nodes by the largest eigenvalues of the adjacency matrices. It turns out that the path $P_{N}$ and star $K_{1, N-1}$ are the trees with the minimum $\lambda_{1}\left(P_{N}\right)$ and maximum $\lambda_{1}\left(K_{1, N-1}\right)$ largest eigenvalues, respectively.

For values $\tau \leq \tau_{c}\left(K_{1, N-1}\right)=\frac{1}{\lambda_{1}\left(K_{1, N-1}\right)}$, it holds that $y_{T}(\tau)=0$, i.e. $J(T)=\alpha(N-1)$ for any tree $T$.

For values $\frac{1}{\lambda_{1}\left(K_{1, N-1}\right)}=\tau_{c}\left(K_{1, N-1}\right)<\tau \leq \tau_{c}\left(P_{N}\right)=$ $\frac{1}{\lambda_{1}\left(P_{N}\right)}$, it holds that $y_{T}(\tau)>y\left(P_{N}\right)=0$, i.e. $J(T)>$ $J\left(P_{N}\right)=\alpha(N-1)$ for any tree $T$. 
Moreover, it is important to mention that although the epidemic threshold $\tau_{c}\left(P_{N}\right)$ of path graph $P_{N}$ is bigger than the epidemic threshold $\tau_{c}\left(K_{1, N-1}\right)$ of star graph $K_{1, N-1}$, function $y(\tau)$ increases faster for $P_{N}$ than $K_{1, N-1}$ in $\tau$.

Theorems 2 and 3 show opposite behavior depending on whether the value $\tau$ is in the high or low regime, although both revolve around the path and star graphs. For $\tau$ in the intermediate regime, we will show that different trees may give the best-/worst-case Nash Equilibrium.

Observation 2. There are values of $\tau$ such that worst-and best-case Nash Equilibria are achieved for trees different from star $K_{1, N-1}$ and path $P_{N}$.

Proof. For example, for $N=5$, there are three nonisomorphic graphs: $K_{1,4}, P_{5}$ and the tree $T^{\prime}$ given in Figure 1c. For $\tau \in[1.475,1.589]$, tree $T^{\prime}$ is the best-case Nash Equilibrium and has optimal social cost.

Theorems 2 and 3 also allow us to compute the Price of Stability (PoS) and the Price of Anarchy (PoA).

Corollary 1. For both high $\left(\tau>\tau_{c}\left(P_{N}\right)\right)$ and low $(\tau \leq$ $\tau_{c}\left(K_{1, N-1}\right)$ ) effective infection rate $\tau$, PoS $=1$, while

$$
\operatorname{PoA}= \begin{cases}1, & \text { for } \tau \leq \tau_{c}\left(K_{1, N-1}\right), \\ \frac{J\left(P_{N}\right)}{J\left(K_{1, N-1}\right)}, & \text { for high enough } \tau>\tau_{c}\left(P_{N}\right)\end{cases}
$$

while PoA $>1$ for $\tau_{c}\left(K_{1, N-1}\right)<\tau \leq \tau_{c}\left(P_{N}\right)$, where PoA $=$ $\infty$ for the special case $\alpha \rightarrow 0$.

Proof. For $\tau>\tau_{c}\left(P_{N}\right)$ (or $\tau \leq \tau_{c}\left(K_{1, N-1}\right)$ ), tree $K_{1, N-1}$ (or $P_{N}$ ) is both optimal in social cost and the best-case Nash Equilibrium. Hence, $\operatorname{PoS}=1$. However, there may be a case in $\tau \in\left[\tau_{c}\left(K_{1, N-1}\right), \tau_{c}\left(P_{N}\right)\right]$, where a tree with minimum $J$ is not a Nash Equilibrium, i.e. $\operatorname{PoS}>1$.

Based on Theorem 2, PoA $=\frac{J\left(P_{N}\right)}{J\left(K_{1, N-1}\right)}$ for big enough $\tau$. Based on Theorem 3, PoA $=1$. For $\tau_{c}\left(K_{1, N-1}\right)<\tau \leq$ $\tau_{c}\left(P_{N}\right), \min J=J\left(P_{N}\right)=\alpha(N-1)$, while star graph $K_{1, N-1}$ is at least one tree (there might be more), where $J\left(K_{1, N-1}\right)>\alpha(N-1)$, hence PoA $>1$. For the special case $\alpha=0$ (no link cost), $\min J=0$, while $J\left(K_{1, N-1}\right)>0$, therefore $\operatorname{PoA}=\infty$. Moreover, PoA is indeed high for such intermediate values of $\tau$ as shown in Fig. 2.

For sufficiently high $\tau$, explicit bounds can be established.

Corollary 2. For sufficiently high effective infection rate $\tau$, in the virus spread-cost game formation,

$$
P o A<1+\frac{1}{2(\tau(\alpha+1)-1)},
$$

where $\tau(\alpha+1)>1$.

Proof. The social cost in star $K_{1, N-1}$ is $J\left(K_{1, N-1}\right)=$ $(N-1) \alpha+\frac{(N-1) \tau^{2}-1}{\tau}\left(\frac{1}{\tau+1}+\frac{1}{(N-1) \tau+1}\right)$, which for high $\tau$ approaches $N+\alpha(N-1)-\frac{(N-1)^{2}+1}{2 \tau(N-1)}$. The social cost of $P_{N}$ is $J\left(P_{N}\right)=\alpha(N-1)+\sum_{i=1}^{N} v_{i \infty}$, where $v_{i \infty}$ are determined by the following recurrent relations $v_{i \infty}=$ $1-\frac{1}{1+\tau\left(v_{(i-1) \infty}+v_{(i+1) \infty}\right)}$, where $v_{i \infty}=v_{(N+1)-i}$ for $i=$
$1, \ldots,\left\lfloor\frac{N+1}{2}\right\rfloor$ and $v_{0 \infty}=v_{(N+1) \infty}=0$. Unfortunately, $v_{i \infty}$ cannot be expressed analytically in an exact, noncanonical, closed form expression. However, for high $\tau$, $J\left(P_{N}\right)$ approaches $N+\alpha(N-1)-\frac{N+2}{2 \tau}$.

For $N=2$, the only feasible graph is $K_{2}$, hence $\operatorname{PoA}=1$. For $N \geq 3$, based on the negligible effect of $O\left(\tau^{-2}\right)$ and using Corollary 1, we arrive at

$\operatorname{PoA}=\frac{N+\alpha(N-1)-\frac{N+2}{2 \tau}}{N+\alpha(N-1)-\frac{(N-1)^{2}+1}{2 \tau(N-1)}}<1+\frac{1}{2(\tau(\alpha+1)-1)}$

The inequality is equivalent to $N \geq \frac{7 \alpha \tau+8 \tau-6}{3 \alpha \tau+4 \tau-3}$, and it holds, because $N \geq 3>\frac{7}{3}>\frac{7 \alpha \tau+8 \tau-6}{3 \alpha \tau+4 \tau-3}$.

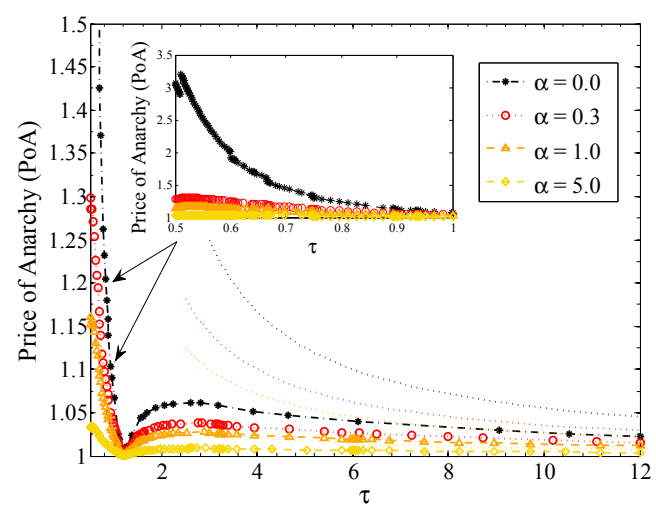

(a) $N=10$.

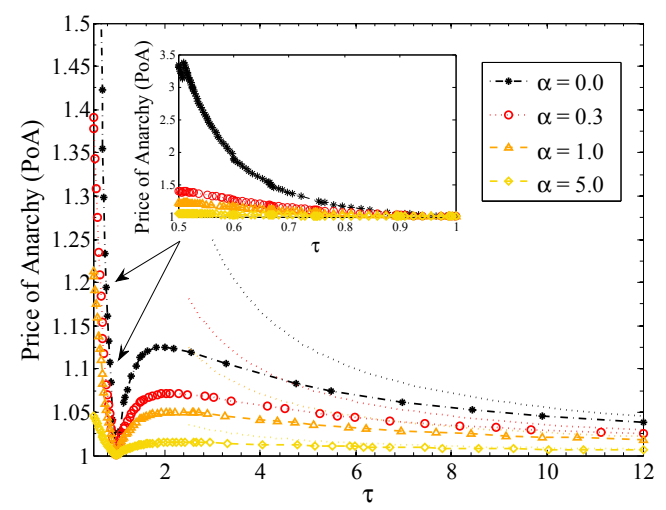

(b) $N=1000$.

Fig. 2: The Price of Anarchy (PoA). The value of $N$ is not of much influence, although higher $N$, in Figure $2 b$, implies more noticeable difference for various $\alpha$. Dotted lines represent the bound from Corollary 2 .

The exact value of the PoA is given in Figure 2 by making use of Corollary 1. The PoA is constant and is larger for smaller installation cost $\alpha$. It is the highest $(\sim 3.3)$ for small $\tau$, above the epidemic threshold, and it further sharply decreases reaching 1 for a unique Nash Equilibrium. For higher $\tau$, the PoA increases reaching its maximum around 1.1 and then it slowly decreases approaching 1 .

We proceed to consider the pairwise Nash Equilibrium and pairwise stability. 
Theorem 4. In the VSC game, a Nash Equilibrium implies pairwise stability and a Nash Equilibrium is equivalent to a pairwise Nash Equilibrium.

Proof. If $G$ is a Nash Equilibrium, then $G$ is a tree and removing a link $l=(i, j)$ by any node $i$ would disconnect the graph, hence $J_{i}(G-(i, j))=\infty$ and $J_{i}(G)<J_{i}(G-(i, j))$. According to Lemma 1, adding a link increases $v_{i \infty}$ and the number of installed links, hence $G$ is pairwise stable and a Nash Equilibrium. Trivially, a pairwise Nash Equilibrium implies that $G$ is also a Nash Equilibrium. However, pairwise stability does not necessarily imply a (pairwise) Nash Equilibrium. Examples are trees that are not Nash Equilibria.

\section{CONCLUSION}

We have considered a novel network formation game (NFG) in relation with security and virus protection in communication networks. In this so-called virus spread-cost VSC game, a player tends to minimize its own cost, which is composed by the virus infection probability and the link installation costs, while maintaining connectivity with the other nodes.

We have characterized the Nash Equilibria and the Price of Anarchy (PoA) in the VSC game. We have found only trees (but not all) could be Nash Equilibria, and surprisingly a path graph is the worst- and the star graph is the best-case Nash Equilibrium and the optimal topology for big virus infection rate $\tau$, while this changes for small and intermediate $\tau$. Moreover, the PoA is the highest for small and intermediate values of $\tau$ just above the epidemic threshold. However, the PoA is generally small, does not depend on the number of players, and is inversely proportional to $\tau$ and the installation cost $\alpha$ for high $\tau$. A PoA close to 1 has design implications: the non-cooperative players' decisions would lead, in a decentralized way, to an optimal topology. Finally, a Nash Equilibrium is pairwise stable.

There are several possibilities for follow-up work, such as a study on mixed Equilibria, player coalitions, inhomogeneous costs weights, or time-varying networks.

\section{ACKNOWLEDGMENTS}

This research has been supported by the EU CONGAS project (project no. 288021).

\section{REFERENCES}

[1] G. Neglia, G. Lo Presti, H. Zang, and D. Towsley, "A network formation game approach to study bittorrent tit-for-tat," in NetgCoop, 2007.

[2] J. Corbo and D. Parkes, "The price of selfish behavior in bilateral network formation," in PODC, pp. 99-107, ACM, 2005.

[3] B.-G. Chun, R. Fonseca, I. Stoica, and J. Kubiatowicz, "Characterizing selfishly constructed overlay routing networks," in INFOCOM, vol. 2, pp. 1329-1339, IEEE, March 2004.

[4] S. Shakkottai, E. Altman, and A. Kumar, "Multihoming of users to access points in wlans: A population game perspective," IEEE Jour. on Sel. Areas in Communications, vol. 25, no. 6, pp. 1207-1215, 2007.

[5] G. Iosifidis, L. Gao, J. Huang, and L. Tassiulas, "Enabling crowdsourced mobile internet access," in INFOCOM, pp. 451-459, IEEE, 2014.

[6] G. Polevoy, S. Trajanovski, and M. M. de Weerdt, "Nash equilibria in shared effort games," in Proc. of AAMAS, (Paris, France), pp. 861-868, ACM, May 2014.
[7] B. Jiang, N. Hegde, L. Massoulie, and D. Towsley, "How to optimally allocate your budget of attention in social networks," in INFOCOM, pp. 2373-2381, IEEE, April 2013.

[8] A. Fabrikant, A. Luthra, E. Maneva, C. H. Papadimitriou, and S. Shenker, "On a network creation game," in PODC, pp. 347-351, ACM, 2003.

[9] R. Aumann and R. Myerson, Endogenous Formation of Links Between Players and of Coalitions: an Application of the Shapley Value. Stanford University, 1988.

[10] P. Van Mieghem, J. Omić, and R. Kooij, "Virus spread in networks," IEEE/ACM Trans. on Netw., vol. 17, pp. 1 -14, feb. 2009.

[11] R. Myerson, Game Theory: Analysis of Conflict. Harvard University Press, 1997.

[12] S. Albers, S. Eilts, E. Even-Dar, Y. Mansour, and L. Roditty, "On Nash Equilibria for a Network Creation Game," in SODA, pp. 89-98, ACM, 2006.

[13] T. Moscibroda, S. Schmid, and R. Wattenhofer, "On the topologies formed by selfish peers," in PODC, pp. 133-142, ACM, 2006.

[14] N. Nisan, T. Roughgarden, E. Tardos, and V. V. Vazirani, Algorithmic Game Theory. Cambridge University Press, 2007.

[15] A. Nahir, A. Orda, and A. Freund, "Topology design and control: A game-theoretic perspective," in INFOCOM, pp. $1620-1628$, april 2009.

[16] N. Alon, E. Demaine, M. Hajiaghayi, and T. Leighton, "Basic network creation games," SIAM Journal on Discrete Mathematics, vol. 27, no. 2, pp. 656-668, 2013.

[17] E. D. Demaine, M. Hajiaghayi, H. Mahini, and M. Zadimoghaddam, "The price of anarchy in network creation games," in PODC, (New York, NY, USA), pp. 292-298, ACM, 2007.

[18] K. Avrachenkov, J. Elias, F. Martignon, G. Neglia, and L. Petrosyan, "A nash bargaining solution for cooperative network formation games," in IFIP Networking, vol. 6640 of LNCS, pp. 307-318, Springer, 2011.

[19] S. Yerramalli, R. Jain, and U. Mitra, "Coalitional games for transmitter cooperation in mimo multiple access channels," IEEE Trans. on Signal Processing, vol. 62, pp. 757-771, Feb 2014.

[20] E. Koutsoupias and C. Papadimitriou, "Worst-case equilibria," Computer Science Review, vol. 3, no. 2, pp. 65 - 69, 2009.

[21] A. Ganesh, L. Massoulie, and D. Towsley, "The effect of network topology on the spread of epidemics," in INFOCOM, vol. 2, pp. 14551466, IEEE, 2005.

[22] D. Chakrabarti, Y. Wang, C. Wang, J. Leskovec, and C. Faloutsos, "Epidemic thresholds in real networks," ACM Trans. Inf. Syst. Secur. vol. 10, pp. 1:1-1:26, Jan. 2008.

[23] D. Shah and T. Zaman, "Detecting sources of computer viruses in networks: Theory and experiment," in SIGMETRICS, (New York, NY, USA), pp. 203-214, ACM, 2010.

[24] P. Van Mieghem, "The N-intertwined SIS epidemic network model," Computing, vol. 93, no. 2-4, pp. 147-169, 2011.

[25] V. M. Preciado, M. Zargham, C. Enyioha, A. Jadbabaie, and G. Pappas, "Optimal vaccine allocation to control epidemic outbreaks in arbitrary networks," in IEEE Conference on Decision and Control (CDC), pp. 7486-7491, Dec 2013.

[26] J. Omić, A. Orda, and P. Van Mieghem, "Protecting against network infections: A game theoretic perspective," in INFOCOM, pp. 1485 1493, IEEE, 2009.

[27] J. Aspnes, K. Chang, and A. Yampolskiy, "Inoculation strategies for victims of viruses and the sum-of-squares partition problem," Jour. of Computer and System Sciences, vol. 72, no. 6, pp. 1077 - 1093, 2006

[28] M. Lelarge and J. Bolot, "Network externalities and the deployment of security features and protocols in the internet," in SIGMETRICS, pp. 37-48, ACM, 2008.

[29] D. Acemoglu, A. Malekian, and A. Ozdaglar, "Network security and contagion," tech. rep., MIT/LIDS intern report, 2013.

[30] Y. Hayel, S. Trajanovski, E. Altman, H. Wang, and P. Van Mieghem, "Complete game-theoretic characterization of sis epidemics protection strategies," in Proc. of CDC (Conference on Decision and Control), (Los Angeles, CA, USA), pp. 1179-1184, IEEE, December 2014.

[31] S. Trajanovski, Y. Hayel, E. Altman, H. Wang, and P. Van Mieghem, "Decentralized Protection Strategies against SIS Epidemics in Networks," IEEE Trans. Control of Network Systems (to appear), 2015.

[32] J. Rosen, "Existence and uniqueness of equilibrium points for concave n-person games," Econometrica, vol. 33, no. 3, pp. 520-534, 1965.

[33] L. Lovász and J. Pelikán, "On the eigenvalues of trees," Periodica Mathematica Hungarica, vol. 3, no. 1-2, pp. 175-182, 1973. 